\title{
Editorial
}

Jerrold H. Levy MD,* Andrew B. Kelly DVm $†$

\section{Inflammation and cardiopulmonary bypass}

Inflammation is a protective response of vascularized tissue normally elicited towards "non-self" determinants or tissue injury, and functions as part of normal host surveillance mechanisms to destroy or quarantine both the harmful agent and the damaged tissue. Most forms of inflammation are exaggerated out of proportion to the stimulus, since humoral amplification systems recruit additional components of the immune system. Activation of inflammatory cascades by foreign material proceeds through a series of integrated steps. In the case of immunological activation, the material to be eliminated (i.e., antigen) is recognized as being "foreign" by immunoglobulins (i.e., antibodies) or by receptors on T-cell-derived lymphocytes that bind to specific determinants. Antigen binding usually leads to activation of an amplification system, initiating production of pro-inflammatory substances called mediators that function to alter blood flow, increase vascular permeability, augment adherence of circulating leukocytes to vascular endothelium, promote migration of leukocytes into tissues, and stimulate leukocytes to destroy the inciting agent. Anaesthetists are familiar with the inflammatory responses that can occur when the immune system "recognizes" drugs, proteins, or tissues as "foreign." Anaphylaxis to anaesthetic drugs, antibiotics, blood products, latex, protamine, or any other agent administered in the perioperative period is the most life-threatening example of an acute inflammatory response. ${ }^{1}$

The actual destruction of antigens by immune mechanisms is mediated by cellular components that migrate freely as polymorphonuclear leukocytes, monocytes, and lymphocytes, or may exist at fixed tissue sites in the form of macrophages. Destruction of antigens usually takes place in tissue spaces and is mediated by polymorphonuclear leukocytes or monocytes, which are recruited from circulating blood. Polymorphonuclear leukocytes (neu-

From the Department of Anesthesiology, Division of Cardiothoracic Anesthesiology \& Critical Care*, and Department of Medicine $\dagger$, Emory University School of Medicine, Atlanta, Georgia. trophils) play a central role in the acute inflammatory phase. In many ways, they are the "hired guns" of the body. Different mechanisms are responsible for directing polymorphonuclear leukocyte accumulation and subsequent activation, central events in the pathogenesis of inflammation.

Cytokines are a class of chemoattractants and white cell activators that are synthesized by activated macrophages to act as secondary messengers and induce the synthesis and expression of specific adhesion molecules on endothelial cells and white cells and that promote attachment and transmigration of leukocytes. ${ }^{2}$ Interleukin1 and tumour necrosis factor are examples of cytokines considered to be the prototype of the pro-inflammatory mediators, and they are important mediators of the biological responses to bacterial lipopolysaccharides, infection, and other inflammatory reactions. Liberation of interleukin-1 and tumour necrosis factor, produces multiple pro-inflammatory effects including fever, neuropeptide release, endothelial cell activation, increased adhesion molecule expression, neutrophil priming, hypotension, myocardial suppression and a catabolic state. ${ }^{2}$

The term interleukin was coined for a group of cytokines that facilitates communication between/among (inter) leukocytes (leukin). Interleukins are a group of different regulatory proteins that act to control many aspects of the immune and inflammatory responses. The interleukins are polypeptides synthesized in response to cellular activation and produce their inflammatory effects by activating specific receptors on inflammatory cells and the vasculature. T-cell lymphocytes influence the activity of other immunological and non-immunological cells by the production of an array of interleukins that they secrete. At least ten different interleukins of this class have been isolated and characterized, and function as short range or intracellular soluble mediators of the immune response and inflammatory responses. The interleukins exert their biological effects by binding to specific polypeptide receptors present on target cell surfaces. The interleukin family of cytokines has been rapidly growing in number due to advances in gene cloning. 
Interleukin-6 is also a cytokine that appears to be under the control of interleukin-1. Interleukin-1 induces synthesis of interleukin- 6 , which is often used as a specific marker for activation of inflammation. ${ }^{3}$ Interleukin-6 is a cytokine with pleiotropic biological activity that is synthesized and secreted by both lymphoid and nonlymphoid cells. ${ }^{4} \mathrm{~T}$ - and B-cell-derived lymphocytes, monocytes, fibroblasts, keratinocytes, endothelial cells, and tumour cells are capable of synthesizing interleukin- $6 .^{5}$ Interleukin-6 is also capable of inducing the acute-phase reactants, and has been used as a marker for induction or suppression of the inflammatory response in patients undergoing cardiopulmonary bypass. ${ }^{6}$

The existence of other types of chemoattractants for polymorphonuclear leukocytes has also been studied. The identification of a novel neutrophil activating factor, distinct from interleukin-1 and tumour necrosis factor, released in large amounts by stimulated mononuclear cells led to the identification of interleukin- $8 .^{7}$ Interleukin-8 has two major effects on human neutrophils, chemotaxis and the release of granule enzymes, and appears to be relatively selective for polymorphonuclear leukocytes. ${ }^{7}$ In human subjects, histological examination following interleukin-8 injection reveals a marked perivascular neutrophil infiltration increasing in intensity over a five-hour period. Most cells were morphologically intact, with little evidence of degranulation and leukocytoclasis changes. ${ }^{8,9}$

In this month's journal, Kawamura et al. investigated myocardial injury determined by increase in creatine phosphokinase, creatine phosphokinase-MB, neutrophils and granulocyte elastase in 11 adult patients undergoing cardiac surgery and cardiopulmonary bypass. ${ }^{10}$ Because interleukin-8 is chemotactic for these cells, it has been suggested that it activates neutrophils. ${ }^{11-13}$ They propose that increases in interleukin- 6 and interleukin- 8 one hour after reperfusion are responsible for neutrophil activation and cell injury. Interleukin-6 also plays an important role in inflammation. Other investigators have also demonstrated elevation of other interleukins following cardiac surgery and cardiopulmonary bypass. ${ }^{6}$ Cytokines, like other inflammatory mediators, have multiple effects on the cardiovascular system. Vascular endothelial activation by cytokines can generate nitric oxide formation which may have direct toxic effects. ${ }^{14}$ Moreover, all the interleukins have the potential to produce toxic effects on the vasculature. Interleukin-6 and interleukin-8 have rich sources of supply from the endothelial cells and are released by hypoxic conditions. However, before neutrophils are activated, they must first adhere to the vascular endothelial cells. Local release of interleukin- 6 and interleukin-8 at the occlusion site could up-regulate adhesion molecules on vascular endothelium. Is interleukin-8 the only potential factor involved in inflammatory in- jury to the heart or other organ system following reperfusion?

Polymorphonuclear leukocytes have been hypothesized to either cause or amplify the process of reperfusion injury in postischaemic myocardium by chemotaxis, activation, and generation of free oxygen radicals, proteolytic enzymes, and other toxic products. ${ }^{15,16}$ Activated cells also release various inflammatory and chemotactic mediators that can result in a cycle of further cellular chemotaxis and injury. ${ }^{17}$ Pathological examination of reperfused myocardial tissue reveals extensive margination of polymorphonuclear leukocytes and capillary plugging of the coronary microvasculature. ${ }^{18-20}$ A complex layering of interrelated events precedes polymorphonuclear leukocyte migration and degranulation. Oversimplified, it follows this pattern: endothelial cells, rendered dysfunctional by prolonged hypoxia, release cytokines which initiate the localized inflammatory response by making the effected endothelial cells and circulating inflammatory cells "sticky," thereby promoting adherence of activated polymorphonuclear leukocytes to activated endothelial cells. ${ }^{21}$ "Trapped" inflammatory cells transmigrate to affected underlying tissues and subsequently degranulate, releasing deleterious oxygen radicals. ${ }^{21}$

One of the primary responses to injury is a pronounced local inflammatory response, in which polymorphonuclear leukocytes and mononuclear cells rapidly accumulate. Our knowledge about how these inflammatory response cells are called to the site of injury has changed dramatically over the past decade. We now know that localization of cells involved in the acute inflammatory response is an orderly, coordinated, usually self-limiting event involving well defined interactions between leukocytes and endothelial cells. ${ }^{22,23}$ In vitro assays and photo cinematography have shown that leukocytes, normally marginate and roll along the vessel endothelium. ${ }^{21}$ During migration, the cells slow, become arrested, and appear adherent. At least three general mechanisms of adhesion have now been defined: (1) rapid transient up-regulation of leukocyte adhesion molecules, (2) rapid transient upregulation of counter receptor adhesion molecules on endothelial cells, and (3) more persistent cytokine activation of endothelial cells, leading to the synthesis of new adhesion molecules. ${ }^{21}$ While resting endothelium lacks molecules that support adhesion, activation of the vascular endothelium by a variety of cytokines, usually locally acting mediators, results in an ordered expression of adhesion molecules on the cell surface. ${ }^{23,24}$ Cytokines, including interleukin-1, interleukin-6, and tumour necrosis factor are the primary signals or internal regulators for the pro-adhesive state. At least five endothelial-leukocyte adhesion molecules have been identified, cloned, and characterized. These include two members of the selectin 
family, E-selectin, P-selectin, and three members of the immunoglobulin gene super family, ICAM-1, ICAM-2, and VCAM-1. ${ }^{11,23-25}$

The associated activation of inflammatory cascades by extracorporeal circuits must also be taken into account when considering inflammatory responses in cardiac surgical patients. During cardiopulmonary bypass, blood interfaces with non-endothelial surfaces of the extracorporeal circuit, and contact activation occurs, generating kallikrein, kinins, complement activation, and a host of cytokines to produce inflammatory responses. ${ }^{26}$ Systemic organ dysfunction, postoperative bleeding, infection, and acute respiratory distress syndrome are complications of cardiopulmonary bypass that are associated with haemolysis, thrombocytopenia, leukopenia, and the occurrence of a whole body inflammatory reaction. ${ }^{27}$ However, when blood contacts the foreign surfaces of the bypass circuit, complex pathways of inflammation and coagulation are initiated to activate platelets, neutrophils, and the contact system of plasma proteases. During cardiopulmonary bypass, activated platelets synthesize thromboxane $A_{2}$, aggregate, release granular contents, and adhere to the extracorporeal circuit. ${ }^{28}$ Cytokines are also generated as part of the whole body inflammatory reaction. ${ }^{6,26,29} \mathrm{Al}-$ though we are able to antagonize the coagulation cascade with systemic heparinization, we are still unable to prevent the associated inflammatory responses associated with cardiopulmonary bypass. The administration of non-specific protease inhibitors that inhibit kallikrein, plasmin, and other aspects of this inflammatory response, have beneficial effects in reducing postoperative bleeding and transfusion requirements. ${ }^{30}$ Preliminary data also suggests these strategies may decrease interleukin- 6 formation. ${ }^{31}$ However, any therapeutic anti-inflammatory strategy, or single isolated marker of inflammation, must be interpreted with some caution because of the interrelationships with other inflammatory cascades.

If a single mediator was in fact responsible for specific inflammatory changes, during cardiopulmonary bypass and reperfusion of the heart, then therapeutic strategies could be developed. ${ }^{32}$ However, to prove whether any of these pathways are important, we need to have measurable effects and specific inhibitors. Currently we can measure most known inflammatory mediators by a variety of commercially available immunoassays, but whether they represent a pivotal role in inflammatory cascades is unknown. Unless a common pathway for the procession of inflammatory events is clear, interventions focusing on one portion of this process, to reverse or impede the process may not be therapeutic. Once inflammatory cascades have been initiated, using specific therapies to block individual limbs of this cascade will probably be ineffective. Earlier and more effective inter- vention, before initiation of inflammatory cascades may play an important role. Because positive and negative feedback systems exist as part of humoral amplification, it is crucial to determine the final common pathway before we can decide what exact role each mediator plays and whether or not antagonism will have any therapeutic effect.

\section{Linflammation et la circulation extracorporelle}

Linflammation est une réaction de défense des tissus vascularisés dirigée normalement vers un agresseur extrinsèque ou une lésion tissulaire. Elle fait partie du processus normal de défense de l'hôte et vise à détruire ou isoler aussi bien l'agresseur que le tissu lésé. Comme des systèmes d'amplification provoquent le recrutement additionnel d'éléments du système immunitaire, la plupart des réponses inflammatoires sont hors de proportion avec le stimulus. L'activation de la cascade inflammatoire par une substance étrangère survient par l'intermédiaire d'une chaîne d'étapes intégrées. Dans le cas de l'activation immunologique, la substance à éliminer (l'antigène) est reconnue comme " étrangère " par les immunoglobines (les anticorps) ou par des récepteurs situés sur les cellules $\mathrm{T}$ lymphocytaires qui se lient à des déterminants spécifiques. La liaison antigénique déclenche ordinairement l'activation d'un système d'amplification. Cette activation initie la production de substances pro-inflammatoires, appelées médiateurs, qui ralentissent le flux sanguin, augmentent la perméabilité vasculaire et l'adhérence des leucocytes circulants, favorisent la migration intra-tissulaire des leucocytes et induisent la destruction du coupable par les leucocytes. Les anesthésistes connaissent la réaction inflammatoire susceptible d'intervenir lorsque le système immunitaire reconnaît comme " étrangers " des drogues, des protéines ou des tissus. L'anaphylaxie aux drogues anesthésiques, aux antibiotiques, aux produits sanguins, au latex, à la protamine ou à tout autre produit administré à la période périopératoire représente un exemple menaçant de la réaction inflammatoire aiguë. ${ }^{\prime}$

La capacité de destruction ponctuelle des antigènes par les mécanismes immunitaires est transmise par les leucocytes, polynucléaires, monocytes et lymphocytes, ou qui migrent en toute liberté et peuvent se fixer sur des sites 
tissulaires fixes sous forme de macrophages. Les polynucléaires neutrophiles jouent un rôle capital dans la phase inflammatoire aiguë. Å plusieurs titres, ce sont les « tueurs à gages" de l'organisme. Différents mécanismes sont responsables pour le contrôle de l'accumulation des polynucléaires et leur activation subséquente, événements primordiaux dans la pathogenèse de linflammation. Les cytokines, synthétisées par les macrophages activés, représentent une classe de chimioattractifs et d'activateurs leucocytaires. Les cytokines jouent le rôle de deuxième messager et induisent la synthèse et l'expression des molécules spécifiques d'adhésion sur les cellules endothéliales et les leucocytes; elle favorisent ainsi l'ancrage et la transmigration des leucocytes. ${ }^{2}$ L'interleukine-1 et le facteur de nécrose tumorale sont deux exemples de cytokines et sont considérées comme les prototypes des médiateurs proinflammatoires. Les cytokines constituent des médiateurs importants de la réponse biologique aux polyliposaccharides bactériens, l'infection et autres réactions inflammatoires. La libération de l'interleukine-1 et du facteur de nécrose tumorale produisent de nombreux effets proinflammatoires dont la fièvre, la libération de neuropeptides, l'activation des cellules endothéliales, l'augmentation de l'expression de la molécule d'adhésion, la sensibilisation des neutrophiles, l'hypotension, l'inhibition myocardique et une état catabolique. ${ }^{2}$

Interleukine est un termes qui désigne cette classe de cytokines qui permettent la communication entre (inter) leucocytes (leukine). Les interleukines constituent un groupe de protéines régulatrices qui contrôlent plusieurs aspects des réponses inflammatoires et immunitaires. Ce sont des polypeptides synthétisées en réponse à l'activation cellulaire. Elles produisent leurs effets anti-inflammatoires par activation de récepteurs spécifiques situés sur la cellule inflammatoire et le système vasculaire. Les cellules lymphocytaires $T$ ont une influence sur l'activité des autres cellules immunitaires et non immunitaires par la variété des interleukines qu'elles sécrètent. De cette classe, au moins dix interleukines différentes ont été isolées et définies. Elles fonctionnent comme des médiateurs à courte portée ou comme des médiateurs intracellulaires solubles de la réponse immunitaire et des réponses inflammatoires. Les interleukines produisent leurs effets biologiques par la liaison à des récepteurs polypeptidiques présents sur la surface des cellules-cibles. Les progrès du cloning génétique ont favorisé la croissance rapide de la famille interleukine des cytokines.

Linterleukine- 6 est aussi une cytokine qui semble contrôlée par l'interleukine-l. Cette dernière induit la synthèse de l'interleukine- 6 utilisée fréquemment comme marqueur spécifique de l'inflammation. ${ }^{3}$ L'interleukine6 est une cytokine avec activité pléitropique biologique, synthétisée et sécrétée aussi bien par les cellules lym- phoïdes que par les cellules non-lymphoïdes. ${ }^{4}$ Les cellules $T$ et $B$ dérivées des lymphocytes, les monocytes, les fibroblastes, les kératinocytes, les cellules endothéliales et tumorales sont capables de synthétiser linterleukine-6. ${ }^{5}$ L'interleukine-6 est aussi capable dinduire des réactifs en phase aiguë et a été utilisée comme marqueur pour l'induction ou la suppression de la réponse inflammatoire chez un patient sous CEC. ${ }^{6}$

D'autres types de chimioattractifs pour polynucléaires ont été aussi étudiés. L'identification d'un nouveau facteur activateur des neutrophiles, distinct de l'interleukine-1 et du facteur de nécrose tumorale, libéré en grandes quantités par les mononucléaires a conduit à l'identification de l'interleukine-8. ${ }^{7}$ L'interleukine-8 a deux effets principaux sur les neutrophiles humains: le chimiotactisme et la libération d'enzymes granulaires; elle semble relativement sélective pour les polynucléaires. ${ }^{7}$ Chez l'humain, après une injection d'interleukine-8, l'histologie révèle une importante infiltration périvasculaire de neutrophiles qui augmente d'intensité sur une période de cinq heures. La plupart des cellules sont morphologiquement intactes et présentent peu d'évidence de dégranulation et de changements leukocytoclasiques. ${ }^{8,9}$

Ce mois-ci, Kawamura et al. étudient la lésion myocardique déterminée par l'augmentation de la créatine phosphokinase, de la créatine phosphokinase M-B, des neutrophiles, de l'élastase granulocytaire chez 11 adultes soumis à une chirurgie cardiaque sous CEC. ${ }^{10}$ Comme l'interleukine-8 est chimiotactique pour les neutrophiles, on a suggéré qu'elle pourrait les activer. ${ }^{11-13}$ Les auteurs émettent l'hypothèse selon laquelle, une heure après la reperfusion, l'augmentation de l'interleukine- 6 et de l'interleukine-8 serait responsable de l'activation des neutrophiles et de la lésion cellulaire. L'interleukine-6 joue aussi un rôle important dans linflammation. D'autres chercheurs ont aussi montré que d'autres interleukines augmentaient après la chirurgie cardiaque et la CEC. ${ }^{6}$ Les cytokines, comme les autres médiateurs de linflammation ont de multiples effets sur le système cardiovasculaire. L'activation de l'endothélium par les cytokines générerait de l'oxyde nitrique qui peut avoir des effets toxiques directs. ${ }^{14}$ De plus, toutes les interleukines ont le potentiel de produire des effets toxiques sur le système vasculaire. L interleukine-6 et l'interleukine-8 sont approvisionnées par les cellules endothéliales et libérées en hypoxie. Cependant, avant d'être activés, les neutrophiles doivent adhérer aux cellules endothéliales vasculaires. $\mathrm{La}$ libération locale d'interleukine-6 et d'interleukine- 8 au site d'occlusion pourrait réguler les molécules d'adhésion sur l'endothélium vasculaire. Linterleukine-8 serait-elle le seul facteur responsable de la lésion inflammatoire cardiaque ou de tout autre système de l'organisme après le reperfusion? 
On croit que les polynucléaires pourraient soit provoquer, soit amplifier le processus de la lésion de reperfusion myocardique post-ischémique par chimiotactisme, activation et regénération de radicaux oxygénés libre, des enzymes protéolytiques et d'autres produits toxiques. ${ }^{15,18}$ Les cellules activées libéreraient aussi des médiateurs inflammatoires et chimiotactiques susceptibles d'initier un cycle supplémentaire de chimiotactisme et de lésion. ${ }^{17}$ L'examen anatomo-pathologique du tissu myocardique reperfusé révèle une margination étendue des polynucléaires et l'obstruction des capillaires coronaires. ${ }^{18-20}$ Une stratification complexe d'événements interdépendants précède la migration et la dégranulation des polynucléaires. De façon très simplifiée, la séquence est la suivante: les cellules endothéliales déréglées par une hypoxie prolongée, libèrent des cytokines qui initient la réponse inflammatoire localisée en rendant les cellules endothéliales et les cellules inflammatoires circulantes « collantes ", favorisant ainsi l'adhésion des polynucléaires activés à des cellules endothéliales activées. ${ }^{21}$ Les cellules inflammatoires capturées transmigrent vers les tissus sous-jacents affectés et, par la suite, perdent leurs granulations en libérant des radicaux oxygénés nocifs. ${ }^{21}$

Face à une lésion, la réaction inflammatoire locale se manifeste principalement par une accumulation rapide des polynucléaires et des mononucléaires. Nos connaissances sur la façon dont ces cellules sont appelées au site de la lésion ont été modifiées drastiquement au cours de la dernière décennie. Nous savons maintenant que la localisation des cellules responsables de la réponse inflammatoire aiguë est un processus méthodique, coordonné, ordinairement autolimitatif nécessitant des interactions bien définies entre leucocytes et cellules endothéliales. ${ }^{22,23}$ Des tests in vitro et la photocinématographie ont montré que les leucocytes s'accolent normalement à la surface interne des vaisseaux lors du ralentissement de la circulation et roulent le long de la paroi endothéliale. ${ }^{21}$ Pendant la migration, la cellule ralentit, s'arrête et adhère. Au moins trois mécanismes d'adhésion sont maintenant définis: 1) régulation transitoire rapide des molécules adhésives leucocytaires, 2) régulation transitoire rapide des molécules des contre-récepteurs adhésifs sur les cellules endothéliales, et 3) activation plus prolongée des cytokines endothéliales conduisant à la synthèse d'une nouvelle molécule adhésive. ${ }^{21}$ Comme l'endothélium au repos ne possède pas les molécules qui supportent l'adhésion, l'activation de l'endothélium vasculaire par une variété de cytokines, ordinairement des médiateurs agissant localement, a pour résultat l'expression ordonnée de molécules adhésives sur la surface cellulaire. ${ }^{23,24}$ Les cytokines, incluant l'interleukine-1, l'interleukine- 6 et le facteur de nécrose tumorale sont les premiers signaux ou régulateurs internes de l'état proad- hésif. Au moins cinq molécules d'adhésion endothéliumleucocyte ont été identifiées, clonées, et caractérisées. Elles comprennent deux membres de la famille sélectine, Esélectine, $\mathbf{P}$-sélectine, et trois membres de la super famille des gênes de l'immunoglobine, ICAM-1, ICAM-2, et VCAM-1. ${ }^{11,23-25}$

Lorsqu'on évalue la réponse inflammatoire du patient de chirurgie cardiaque, on doit aussi tenir comte de l'activation associée des cascades inflammatoires. Pendant la CEC, le sang entre en contact avec les surfaces nonendothéliales du circuit extracorporel. Une activation par contact intervient, avec génération de kallicréine, kinines, coactivation du complément; une armée de cytokines se mobilise pour produire les réponses inflammatoires. ${ }^{28}$ Le dérèglement systémique, le saignement postopératoire, linfection et le syndrome de détresse respiratoire aigu sont des complications de la CEC. Elles s'associent à l'hémolyse, la thrombocytopénie, la leukopénie et l'apparition d'une réaction inflammatoire généralisée. ${ }^{27}$ Le contact du sang avec.la surface du circuit extracorporel, les chaines complexes de l'inflammation et de la coagulation sont déclenchées avec activation des plaquettes, des neutrophiles, et du système de contact des protéase plasmatiques. Pendant la CEC, les plaquettes activées synthétisent la thromboxane $A_{2}$, s'agrégent, libèrent leur contenu granuleux et adhérent au circuit extracorporel. ${ }^{28}$ Les cytokines qui font partie de la réaction inflammatoire globale sont aussi générées. ${ }^{6,26,29}$ Bien que nous soyons en mesure d'antagoniser la cascade de la coagulation par l'héparinisation systémique, nous sommes toujours impuissants à prévenir les réponses inflammatoires associées à la CEC. L'administration d'inhibiteurs non spécifiques de la protéase qui inhibent la kallikréine, la plasmine et autres éléments de la réponse inflammatoire, produit des effets bénéfiques sur le saignement postopératoire et les besoins transfusionnels. ${ }^{30}$ Des données préliminaires suggèrent aussi que ces mesures pourraient réduire la formation d'interleukine- $6{ }^{31}$ Cependant, toute thérapeutique anti-inflammatoire, ou tout marqueur isolé de l'inflammation doivent être analysés avec prudence à cause de l'inter-relation entre plusieurs cascades inflammatoires.

En effet, si un simple médiateur était responsable de changements inflammatoires spécifiques pendant la CEC et la reperfusion cardique, on pourrait certes planifier des thérapies. ${ }^{32}$ Cependant, pour prouver l'importance d'une des chaînes, nous avons besoins d'en connaître les effets mesurables et les inhibiteurs spécifiques. Présentement, grâce aux immuno-essais du commerce, nous pouvons mesurer la plupart des médiateurs inflammatoires connus, mais l'importance de leur rôle sur les cascades inflammatoires est inconnue. A moins qu'il n'existe une chaîne commune pour le déroulement des événements, les interventions visant à renverser ou à bloquer une par- 
tie du processus n'auront aucune valeur thérapeutique. Une fois une cascade inflammatoire amorcée, l'utilisation de thérapies spécifiques pour en bloquer un affluent est vaine. Une intervention plus précoce pourrait être plus efficace. Comme l'amplification humorale comporte des systèmes positifs et négatifs de rétroaction, il est crucial de connaître la chaîne terminale commune avant de pouvoir décider du rôle exact de chaque médiateur et de déterminer quel antagoniste, s'il en est, aurait un effet thérapeutique.

\section{References}

I Levy JH. Anaphylactic Reactions in Anesthesia and Intensive Care. 2nd ed. Boston: Butterworth-Heinemann, 1992.

2 Pober JS, Cotran RS. Cytokines and endothelial cell biology. Physiol Rev 1990; 70: 427-51.

3 Loppnow H, Libby P. Proliferating or interleukin 1activated human vascular smooth muscle celles secrete copious interleukin 6. J Clin Invest 1990; 85: 731-8.

4 Wong G. Clark SC. Multiple actions of interleukin-6 within a cytokine network. Immunology Today 1988; 9: 137-9.

5 Hirano T, Kishimoto T. Interleukin-6. In: Sporn MB, Roberts AB. (Eds.). Peptide growth factors and their receptors. Berlin: Springer-Verlag: 1990; 633-65.

6 Sakai T, Latson TW, Whitten CW, Ring WS, Lipton JM. Perioperative measurements of interleukin 6 and alphamelanocyte-stimulating hormone in cardiac transplant patients. J Cardiothor Vasc Anesth 1993; 7: 17-22.

7 Yoshimura T, Matsushima K, Tanaka S. Purification of a human monocyte-derived neutrophil chemotactic factor that has peptide sequence similarity to other host defense cytokines. Proc Natl Acad Sci U S A 1987; 84: 9233-7.

8 Leonard EJ, Yoshimura T, Tanaka S, Raffeld M. Neutrophil recruitment by intradermally injected neutrophil attractant/activation protein 1. J Invest Dermatol 1991; 96: 690-4.

9 Swensson O, Schubert C, Christophers E, Schroder JM. Inflammatory properties of neutrophil-activating protein-1/ interleukin 8 (NAP-1/IL-8) in human skin: a light and electron microscopic study. J Invest Dermatol 1991; 96: 682-9.

10 Kawamura T, Inada S, Koyama H, Okada K, Wakusawa $R$. The elevation of cytokines in open heart surgery with cardiopulmonary bypass: participation of interleukin-8 and 6 to reperfusion injury. Can J Anesth 1993: 41: 1016-21.

11 Pober JS, Cotran RS. The role of endothelial cells in inflammation. Transplantation 1990; 50: 537-44.

12 Schroder JM, Mrowietz U, Morita E, Christophers E. Purification and partial biochemical characterization of a human monocyte-derived neutrophil-activating peptide that lacks interleukin-1 activity. J Immunol 1987; 139: 3474-83.
13 Peveri P, Walz A, Dewald B, Baggiolini M. A novel neutrophil-activating factor produced by human mononuclear phagocytes. J Exp Med 1988; 167: 1547-59.

14 Finkel MS, Oddis CV, Jacob TD, Watkins SC, Hattler BG, Simmons $R L$. Negative inotropic effects of cytokines on the heart mediated by nitric oxide. Science 1992; 257: 387-9.

15 Shandelya SML, Kuppusamy P, Weisfeldt ML, Zweier JL. Evaluation of the role of polymorphonuclear leukocytes on contractile function in myocardial reperfusion injury. Evidence for plasma-mediated leukocyte activation. Circulation 1993; 87: 536-46.

16 Romson JL, Hook BG, Kunkel SL, Abrams GD, Schork $M A$, Lucchesi $B R$. Reduction of the extent of ischemic myocardial injury by neutrophil depletion in the dog. Circulation 1983; 67: 1016-23.

17 Rowe GT, Eaton LR, Hess ML. Neutrophil-derived, oxygen free radical-mediated cardiovascular dysfunction. J Mol Cell Cardiol 1984; 16: 1075-9.

18 Engler $R$. Consequences of activation and adenosinemediated inhibition of granulocyte during myocardial ischemia. Fed Proc 1978; 46: 2407-12.

19 Engler RL, Schmid-Schonbein GW, Pavelel RS. Leukocyte capillary plugging in myocardial ischemia and reperfusion in the dog. Am J Pathol 1983; 111 : 98-111.

20 Mehta JL, Nichols WW, Donnelly WH, et al. Protection by superoxide dismutase from myocardial dysfunction and attenuation of vasodilator reserve after coronary occlusion and reperfusion in dogs. Circ Res 1989; 65: 1283-95.

21 Harlan JM, Liu DY. Adhesion: Its Role in Inflammatory Disease. First ed. New York: W.H. Freeman and Company, 1992.

22 Dzau VJ, Gibbons GH, Cooke JP, Omoigui N. Vascular biology and medicine in the 1990s: scope, concepts, potentials, and perspectives. Circulation 1993; 87: 705-19.

23 Bevilacqua MP, Nelson RM. Endothelial-leukocyte adhesion molecules in inflammation and metastasis. Thromb Haemost 1993; 70: 152-4.

24 Bevilacqua MP, Pober JS, Wheeler ME, Mendrick D, Cotran RS, Gibrone MA. Interleukin-1 acts on cultured human vascular endothelial cells to increase the adhesion of polymorphonuclear leukocytes, monocytes, and related leukocyte cell lines. J Clin Invest 1985; 76: 2003-11.

25 Kappelmayer J, Bernabei A, Gikakis N, Edmunds LH Jr, Colman RW. Upregulation of Mac-1 surface expression on neutrophils during simulated extracorporeal circulation. $J$ Lab Clin Med 1993; 121: 118-26.

26 Butler J, Chong GL, Baigrie RJ, Pillai R, Westaby S, Rocker $G M$. Cytokine responses to cardiopulmonary bypass with membrane and bubble oxygenation. Ann Thorac Surg 1992; 53: 833-8.

27 Westaby $S$. Organ dysfunction after cardiopulmonary by- 
pass. A systemic inflammatory reaction initiated by the extracorporeal circuit. Intensive Care Med 1987; 13: 89-95.

28 Woodman $R C$, Harker LA. Bleeding complications associated with cardiopulmonary bypass. Blood 1990; 76 : 1680-9.

29 Haeffner-Cavaillon N, Roussellier N, Ponzio O, et al. Induction of interleukin-1 production in patients undergoing cardiopulmonary bypass. J Thorac Cardiovasc Surg 1989; 98: 1100-6.

30 Royston $D$. High dose aprotinin therapy: a review of the first five years' experience. J Cardiothor Vasc Anesth 1992; 6: $76-100$.

31 Whitten $C W$, Latson TW, Allison PM, et al. Does aprotinin inhibit cardiopulmonary bypass-induced inflammation? Anesthesiology 1992; 77: A266.

32 Dinarello CA, Gelfand JA, Wolff SM. Anticytokine strategies in the treatment of the systemic inflammatory response syndrome. JAMA 1993; 269: 1829-35. 